\title{
Review
}

\section{Sympathetic Overdrive and Cardiovascular Risk in the Metabolic Syndrome}

\author{
Guido GRASSI ${ }^{1-3)}$
}

\begin{abstract}
Sympathetic neural factors are involved in energy balance as well as in blood pressure control. This represents the background for the hypothesis that an adrenergic overdrive may be implicated in the development and/or progression of the metabolic syndrome. Indirect and direct markers of sympathetic drive have confirmed this hypothesis, by showing the occurrence of an adrenergic activation both at the cardiac and peripheral vascular level. It is likely that this sympathetic dysfunction is triggered by reflex mechanisms (arterial baroreceptor impairment), metabolic factors (insulin resistance), and humoral agents (angiotensin II, leptin). The adrenergic overdrive exerts a number of adverse effects on the cardiovascular system, by favoring the genesis of cardiac hypertrophy, vascular hypertrophy, arterial remodeling and endothelial dysfunction and thereby aggravating the already elevated cardiovascular risk profile of the patient. This carries obvious clinical and therapeutic implications, including the suggestion that sympathetic inhibition should be included among the goals of both pharmacological and non-pharmacological interventions employed in the treatment of the metabolic syndrome. (Hypertens Res 2006; 29: 839-847)
\end{abstract}

Key Words: metabolic syndrome, sympathetic nervous system, hypertension, obesity, antihypertensive drugs

\section{Introduction}

During the past few years, a condition characterized by the clustering of several cardiovascular and metabolic risk factors, known as "metabolic syndrome," has engendered growing interest among clinicians and investigators due to its unfavorable impact on cardiovascular morbidity and mortality. However, because there is no comprehensive picture of the disease pathophysiology, no specific guidelines have been developed for therapeutic intervention.

The present paper will critically review the hypothesis that a dysfunction in the sympathetic control of the cardiovascular and metabolic function might be responsible for the disease pathophysiology and the related cardiovascular risk, thus representing a specific target of the therapeutic intervention. I will first consider the sympathetic alterations occurring under specific conditions clustering in the metabolic syndrome, such as hypertension and obesity. I will then examine the role played by neurogenic factors in the metabolic syndrome and the metabolic, reflex and humoral mechanisms potentially responsible for the profound neuroadrenergic abnormalities detectable in this condition. I will also examine the participation of the sympathetic nervous system in the development and/or progression of the cardiac, vascular and renal endorgan damage frequently observed in the clinical course of the disease. This will allow me to address, in the final part of the paper, the therapeutic implications of the above-mentioned findings and to discuss the treatment options based on drugs capable of inhibiting the sympathetic neural drive and thereby ameliorating the adverse cardiometabolic effects of the disease.

From the ${ }^{1}$ Istituto di Clinica Medica, Dipartimento di Medicina Clinica, Prevenzione e Biotecnologie Sanitarie, Università Milano-Bicocca, Ospedale San Gerardo, Monza (Milan), Italy; ${ }^{2)}$ Istituto Auxologico Italiano, Milan, Italy; and ${ }^{3)}$ Centro Interuniversitario di Fisiologia Clinica e Ipertensione, Università di Milano, Milano-Bicocca, Italy.

Address for Reprints: Guido Grassi, M.D., Clinica Medica, Ospedale San Gerardo, Via Pergolesi 33, 20052, Monza (Milan), Italy. E-mail: guido.grassi@unimib.it

Received May 8, 2006; Accepted in revised form July 19, 2006. 


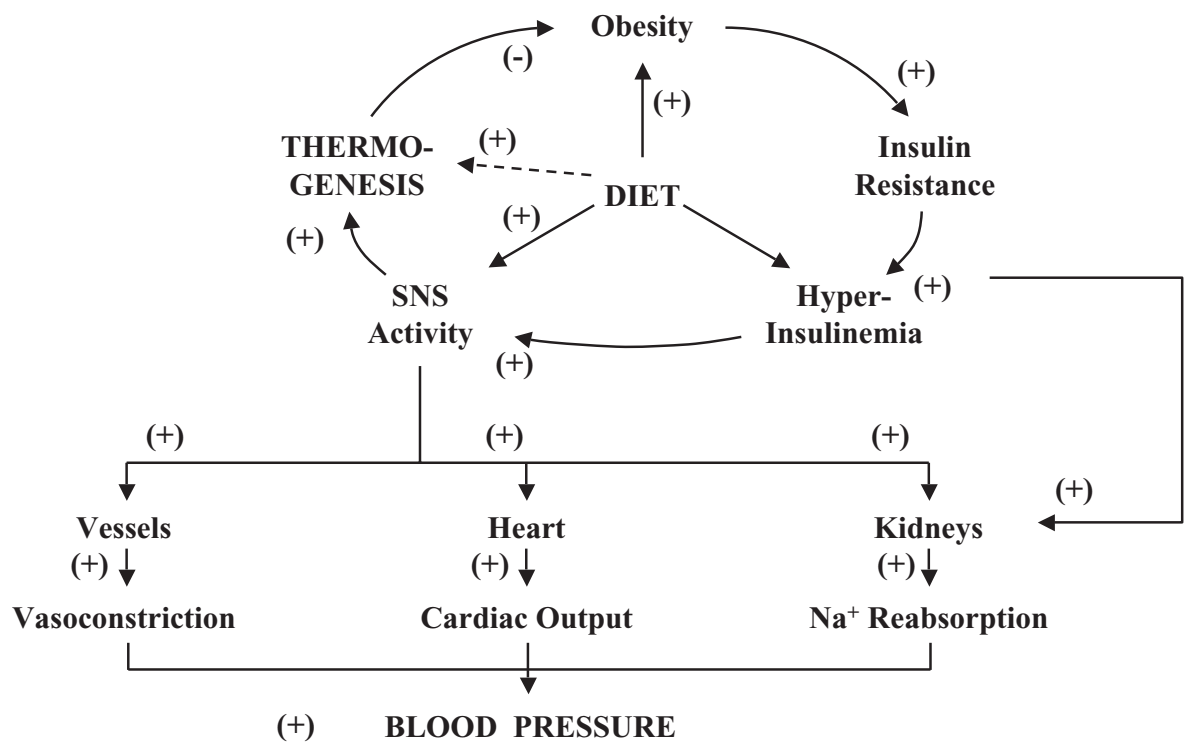

Fig. 1. Scheme illustrating the role exerted by the Sympathetic Nervous System (SNS) in energy balance (top) and blood pressure (bottom) control. Symbols (+) and (-) refer to excitatory or inhibitory effects. Modified from Reaven et al. (1).

\section{Sympathetic Function in Hypertension and in Obesity}

The hypothesis that a functional alteration in the sympathetic nervous system might represent the pathophysiologic "core" of the metabolic syndrome is based on evidence that the two main variables which appear to be primitively deranged in this condition, i.e., blood pressure and thermogenesis, are under adrenergic influences (Fig. 1). Indeed, evidence has been provided that, in experimental animals, alterations in sympathetic control of energy expenditure may favor directly or indirectly (i.e., without or with the presence of an insulin resistance state) a gain in body weight and, with time, the occurrence of an overweight or obese state $(1,2)$. On the other hand, data collected in experimental animal models of hypertension as well as in human hypertension provide conclusive evidence that alterations in the sympathetic control of heart rate, cardiac output, peripheral vascular resistance and renal sodium handling may promote, alone or in combination, the development and progression of the hypertensive state (3-5).

As far as essential hypertension is concerned, a consistent number of direct and indirect evidences provide almost univocal support of the hypothesis that high blood pressure states are characterized by sympathetic overactivity. For example "old" data collected in the Tecumseh Study have shown that young patients with borderline hypertension (and particularly those with a hyperkinetic circulation) display a resting tachycardia associated with increased plasma norepinephrine values (6). Since heart rate values 1) depend on both vagal and sympathetic neural influences (7) and 2) correlate with other independent markers of adrenergic drive (8), the conclusion has been drawn that early hypertensive phases are already characterized by a hyperadrenergic state. In more recent years, techniques based on direct assessment of sympathetic tone, such as the microneurographic recording of efferent sympathetic nerve traffic to the skeletal muscle district and the so-called "norepinephrine radiolabeled technique" (9), have supported these indirect evidences, expanding the information available on the behavior of the sympathetic function in early hypertensive phases to the stable and/or more severe forms of the disease. This is shown in Fig. 2, which refers to the results of a microneurographic study performed by our group in mild and more severe essential hypertensive patients (10). Indeed, when the nerve firing rate, i.e., the number of sympathetic bursts over time or corrected for heart rate, was assessed in normotensive middle-age subjects and in untreated age-matched patients displaying blood pressure elevations of increasing severity, the results showed that not only mild but also more severe essential hypertension was characterized by sympathetic overactivity. Such findings indicate that the degree of adrenergic activation is directly proportional to the severity of the hypertensive state.

The microneurographic approach has also led to three important findings regarding the participation of neurogenic mechanisms in the development of hypertension. First, the sympathetic overactivity appears to be a specific feature of the essential hypertensive state, since no adrenergic overdrive is reported in secondary forms of hypertension, such as in renovascular hypertension or in hyperaldosteronism (Fig. 2) (10). Second, not only young or middle-age patients but also elderly subjects with isolated systolic or systodiastolic hypertension display an increased sympathetic nerve-firing rate (11). Finally, as discussed below, the magnitude of the sym- 
MAP

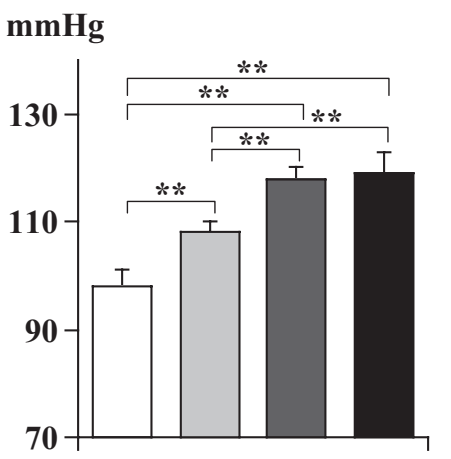

HR

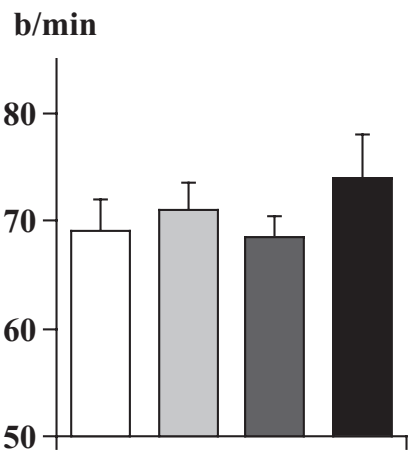

MSNA

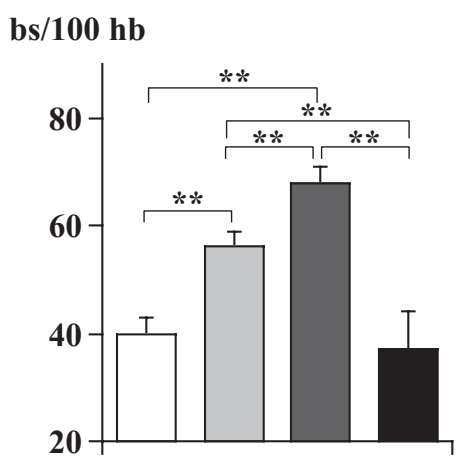

$\square$ NT $(n=15) \quad \square$ Moderate EH $(n=14) \quad \square$ More Severe EH $(n=14) \quad \square$ Secondary HT $(n=13)$

Fig. 2. Mean arterial pressure (MAP), heart rate (HR) and muscle sympathetic nerve traffic (MSNA) in normotensive subjects (NT) and in patients with moderate and more severe essential hypertension (EH). Data in secondary hypertension (HT) are also shown. Data are shown as the means \pm SEM. Asterisks indicate statistically significant differences between groups ( ${ }^{*} \mathrm{p}<0.05$, $* * \mathrm{p}<0.01)$. This figure is based on data from Grassi et al. (10).

pathetic activation appears to be heightened when the hypertensive state is complicated by cardiac hypertrophy, i.e., when target organ damage is present $(12,13)$.

The norepinephrine spillover technique has provided further information on the behavior of adrenergic function in hypertension. In this method, small amounts of tritiated norepinephrine and epinephrine are infused into humans, thereby allowing quantification of the spillover and clearance rate of the adrenergic neurotransmitters in both the systemic and regional circulation (9). This approach has demonstrated that there is an "actual" increase in the secretion of norepinephrine from sympathetic nerve terminals in hypertension, and that this increase occurs in organs displaying a specific pro-hypertensive pathogenetic role, such as the kidney, or in districts exposed to the complications of the hypertensive state, such as the cerebral and the coronary circulation $(14,15)$.

Unlike in hypertension, in obesity the interest of investigators has only recently focused on the behavior of sympathetic function. However, a wide range of information has already been obtained, and can be summarized as follows. The obese state, even when unaccompanied by a blood pressure elevation, displays signs of adrenergic activation, such as increased resting heart rate values and elevated plasma norepinephrine values (16). This has been confirmed by the results of the Normative Aging Study, which in a large number of subjects has shown a close direct relationship between 24-h urinary figures of norepinephrine metabolites and body mass index values (17). This has also been confirmed by the evidence that an augmented sympathetic neural discharge to skeletal muscle as well as an increased spillover rate of norepinephrine from sympathetic nerve endings, particularly at the level of the kidney, takes place in obese normotensive subjects $(18$,
19). Taken together, these findings provide conclusive evidence that the sympathetic overdrive plays a role in human obesity, although the magnitude of this role differs somewhat according to the specific patterns of fat distribution. This is shown in Fig. 3, which displays the results of a recent microneurographic study in which the muscle sympathetic neural drive was assessed in abdominal as well as in peripheral obesity (20). The results clearly indicate that the degree of sympathetic activation (and the magnitude of the insulin resistance state) is much greater in patients with visceral body fat deposits than in those with peripheral distribution of the adipose tissue. This finding (which, as discussed below, underscores once again the close relationships between sympathetic overactivity and insulin sensitivity) has been further confirmed by the results of a study showing that subcutaneous body fat accumulation is not linked to an adrenergic overdrive (21).

Should the sympathetic overactivity be regarded as a feature common to almost all obese states or rather to specific clinical conditions? The question, which has been advanced in the past few years, does not appear to be an "academic" one, since a state of adrenergic overdrive carries an adverse impact on cardiovascular morbidity and mortality, thus representing an important clinical and therapeutic target. The background for this question was provided by a study suggesting that obesity results in a hyperadrenergic state only when it is associated with obstructive sleep apnea (22). The question was recently re-examined by our group in a study in which microneurographic assessment of muscle sympathetic nerve traffic was performed in lean and obese subjects with or without polysomnographic evidence of sleep apnea (23). The study's results, summarized in Fig. 4, allow us to draw three main conclusions. First, human obesity, even when uncou- 

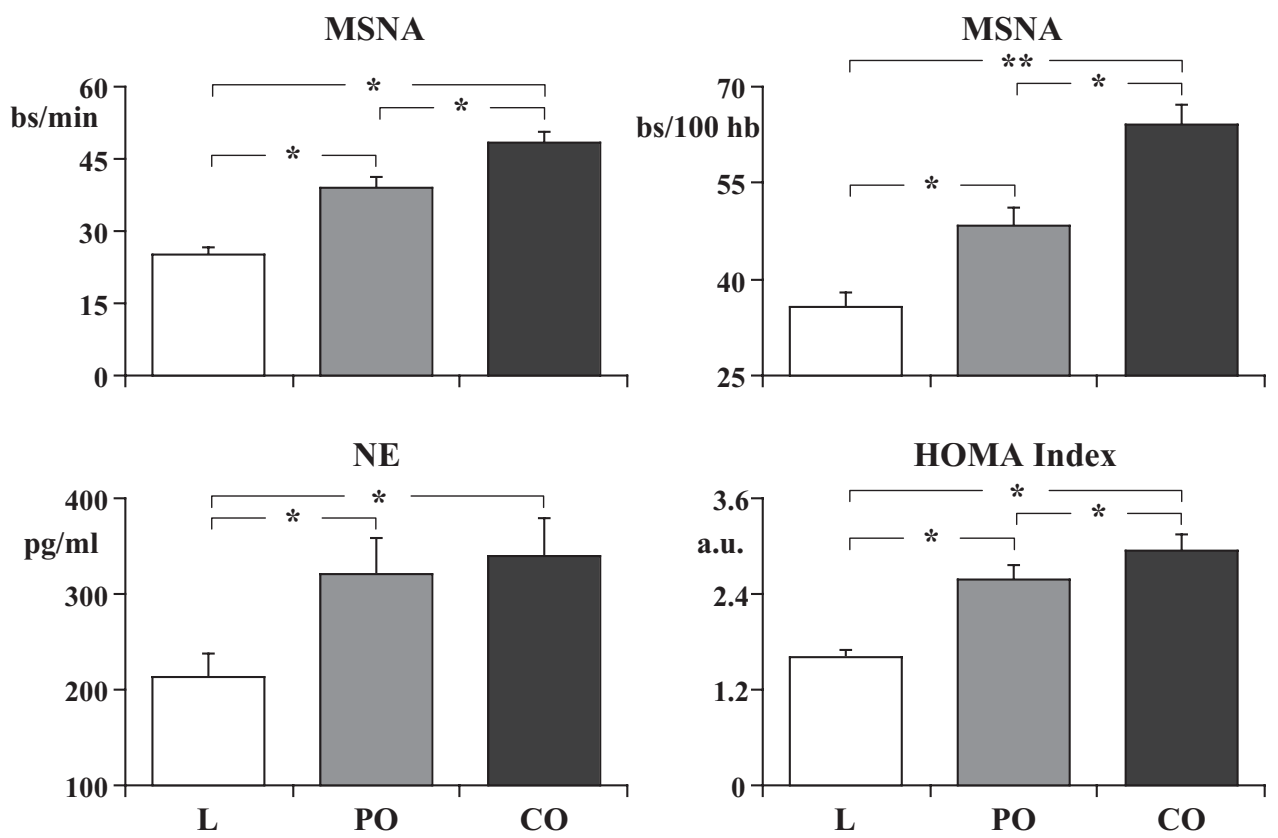

Fig. 3. Muscle sympathetic nerve traffic (MSNA), plasma norepinephrine (NE) and HOMA index values in lean subjects (L) and in patients with peripheral (PO) and central (CO) adiposity. Data are shown as the means \pm SEM. MSNA data are expressed both as bursts incidence over time (bs/min) and as number of bursts corrected for heart rate (bs/100 hb). Asterisks indicate statistically significant differences between groups $\left({ }^{*} \mathrm{p}<0.05,{ }^{*} \mathrm{p}<0.01\right)$. This figure is based on data from Grassi et al. (20).

pled with sleep apnea, induces a hyperadrenergic overdrive of the cardiovascular system. Second, sleep apnea exerts potentiating effects on the sympathetic function, increasing by 30 $40 \%$ the adrenergic overdrive seen in obese subjects without this complication. Finally, the sympathoexcitatory effects triggered by sleep apnea do not seem peculiar to the obese state, their presence being also detectable in normoweight individuals. It thus appears that the sympathetic overdrive characterizing the obese state is not limited to patients with sleep apnea.

\section{Sympathetic Overdrive in the Metabolic Syndrome}

As mentioned above, there is a solid background to the hypothesis that neurogenic alterations occur in metabolic syndrome. This is based on the evidence that 1) cardiovascular as well as metabolic function are modulated by sympathetic mechanisms and 2) several of the pathologic states clustering in the metabolic syndrome already display an increased adrenergic drive $(2,3,10,18-20)$. This is also based on the finding that when obesity and hypertension are concomitantly present in the same patient, the degree of sympathetic activation is much greater than in patients with either condition singly (24).

Data collected on this issue in recent years provide evidence that sympathetic overdrive does occur in metabolic syndrome. First, data based on urinary normetanephrine assay have demonstrated an increase in 24-h excretion of this catecholaminergic metabolite in patients with metabolic syndrome (25). Second, a reduced low frequency/high frequency power, suggestive of a hyperadrenergic drive, has been reported in patients with, but not in those without, the clustering of metabolic risk factors (25). Third, whole body norepinephrine spillover appears to be reduced in patients with metabolic syndrome who undergo lifestyle interventions (diet, physical exercise) capable of improving and/or reversing the single components of the disease (26). Finally, two recent microneurographic studies have independently shown that middle-age subjects with metabolic syndrome display greater levels of sympathetic nerve traffic $(27,28)$. Interestingly, the sympathetic overdrive appears to be independent of the presence of hypertension, since it is detectable even in patients without high blood pressure (Fig. 5). It also appears to be detectable in cardiovascular diseases that are frequently characterized by sympathetic activation, such as in heart failure complicated by metabolic syndrome (29). Taken together, these findings suggest that the metabolic syndrome-dependent sympathoexcitation is a common finding in uncomplicated or complicated clinical states.

\section{Mechanisms Responsible for the Sympathetic Abnormalities in the Metabolic Syndrome}

Long-term changes in sympathetic drive, such as those 

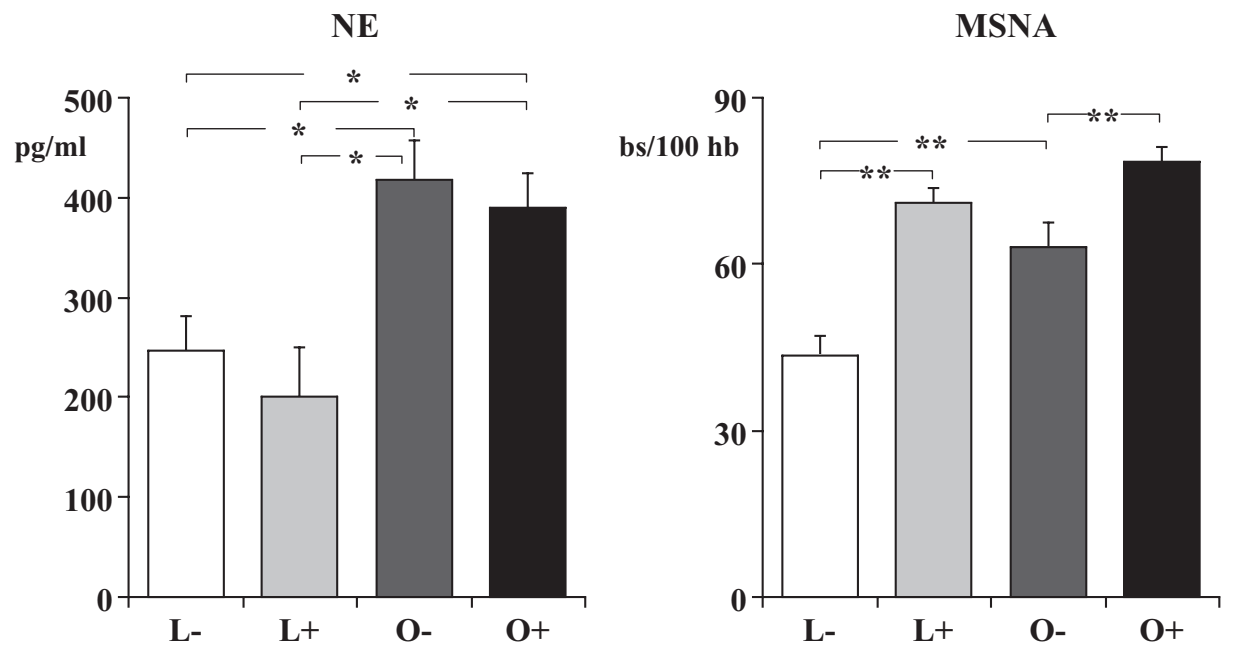

Fig. 4. Plasma norepinephrine (NE) and muscle sympathetic nerve traffic (MSNA) values detected in lean subjects without ( $L-$ ) and with $(\mathrm{L}+)$ sleep apnea and in age-matched obese patients without $(\mathrm{O}-)$ and with $(\mathrm{O}+)$ sleep apnea. Data are shown as the means \pm SEM. Asterisks indicate statistically significant differences between groups $\left({ }^{*} \mathrm{p}<0.05, * * \mathrm{p}<0.01\right)$. Note that obese subjects display sympathetic activation even when the obese state is not complicated by sleep apnea. This figure is based on data from Grassi et al. (23).

MSNA

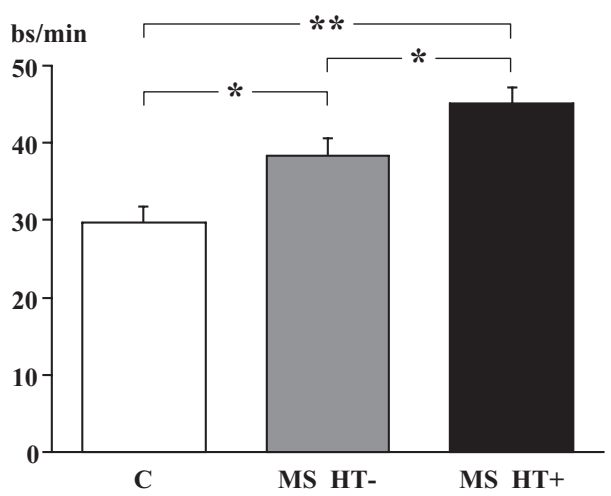

MSNA

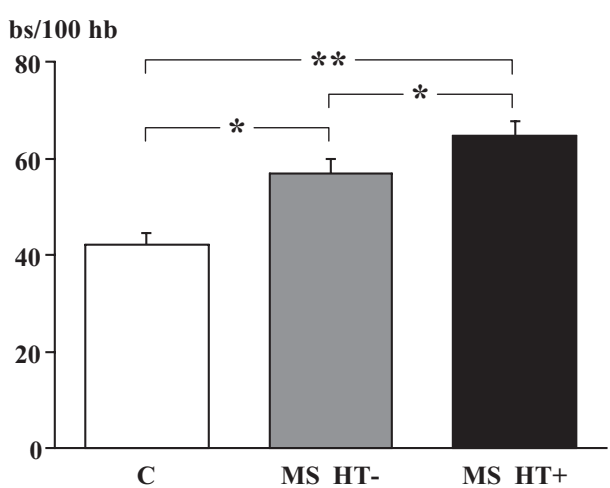

Fig. 5. Muscle sympathetic nerve traffic values in control eumetabolic subjects (C) and in normotensive (MS HT-) and hypertensive (MS HT+) patients with metabolic syndrome. Data are shown as the means \pm SEM. Asterisks indicate statistically significant differences between groups $\left({ }^{*} \mathrm{p}<0.05,{ }^{* *} \mathrm{p}<0.01\right)$. Note that patients with metabolic syndrome display sympathetic activation even when hypertensive patients are excluded from data analysis. This figure is based on data from Grassi et al. (28).

described in the metabolic syndrome and in its single components, are likely to have a multiple physiopathological nature. One of the hypotheses advanced is that the neurogenic alterations originate from an impairment of the baroreflex, i.e., a major restraining mechanism on sympathetic tone (3). This possibility appears to be confirmed by the evidence that arterial baroreceptor control of sympathetic nerve traffic undergoes a clearcut impairment in patients with metabolic syndrome and that both the sympathoinhibitory and the sympathoexcitatory baroreflex components are involved (28). The reflex impairment may also involve other reflexogenic areas, such as the cardiopulmonary receptors and the chemoreceptors as well (3). Although no specific data are available on the behavior of these reflex functions in the metabolic syndrome, data collected in hypertensive patients with left ventricular hypertrophy or in obese subjects with sleep apnea syndrome do provide clearcut evidence that alterations in the reflex restraint exerted by these reflexogenic areas on adrenergic drive may participate in the occurrence of the hyperadrenergic state $(30,31)$.

It has also been hypothesized that the sympathetic overactivity occurring in metabolic syndrome is dependent on the hyperinsulinemia and the related insulin resistance state characterizing the disease (32). This hypothesis comes from the 
Table 1. Effects of Different Antihypertensive Drug Classes on Sympathetic Function on Insulin Profile

\begin{tabular}{lcc}
\hline \multicolumn{1}{c}{ Drug class } & $\begin{array}{c}\text { Sympathetic } \\
\text { activity }\end{array}$ & $\begin{array}{c}\text { Insulin } \\
\text { resistance }\end{array}$ \\
\hline Diuretics & Increased & Worsened \\
$\beta$-Blockers & Decreased & Worsened \\
Calcium antagonists & Unchanged & Unchanged \\
ACE inhibitors & Decreased & Improved \\
Ang II receptor blockers & Decreased & Improved \\
Central sympatholytics & & \\
\multicolumn{1}{c}{$\left(\mathrm{I}_{1}\right.$ agonists) } & Decreased & Improved
\end{tabular}

ACE, angiotensin-converting enzyme; Ang II, angiotensin II.

evidence that, in experimental animals as well as in humans, acute systemic administration of insulin, performed without affecting glucose levels in the context of the so-called euglygemic clamp approach, triggers a marked sympathetic stimulation (32-35). Several data further support the abovementioned hypothesis. For example, it has been shown that the level of activation of the sympathetic nervous system and the level of insulin resistance are closely correlated in both cardiovascular and non-cardiovascular diseases. Furthermore, recent data by our group suggest that, in obese subjects not yet displaying an insulin resistance state, the degree of sympathetic activation is much less than that detected in agematched obese individuals with reduced insulin sensitivity (36). Finally, as discussed below, the effects of antihypertensive drugs on adrenergic drive (sympathoexcitation or sympathoinhibition) are frequently mirrored by their impact on insulin sensitivity (worsening or improvement) (Table 1). A further intriguing finding is that the sympathetic-insulin crosstalks are bidirectional, and at present it is still unknown whether the sympathetic activation is responsible for the insulin resistance state or whether it represents an epiphenomenon of the metabolic alteration $(32,33,37)$.

A final possibility is that the sympathetic overactivity depends on the hormonal factors involved in the homeostatic control of the cardiovascular function. They include, for example, angiotensin II, which 1) enhances neuroadrenergic cardiovascular drive through stimulation of receptors located within the hypothalamus (paraventricular nucleus) (38), 2) interacts with the nitric oxide system to potentiate sympathetic function $(38,39)$ and 3$)$ affects norepinephrine turnover at the level of peripheral nerve endings (40). This hypothesis appears to be confirmed also in humans by the evidence that in obese hypertensive patients, patients with renal insufficiency, or patients with left ventricular failure (i.e., in conditions characterized by a marked sympathetic and reninangiotensin activation), administration of angiotensin II receptor blockers results in a clearcut sympathoinhibition (41-43). A further hormonal substance is leptin, which acts at the level of the hypothalamus to increase blood pressure via its central sympathoexcitatory effects $(44,45)$.

\section{Sympathetic Activation, Cardiovascular Risk and Organ Damage}

In several pathological conditions there is a close link between sympathetic activity and cardiovascular risk. This has been shown, for example, in patients with congestive heart failure, in which indirect and direct markers of systemic and regional sympathetic drive (venous plasma norepinephrine, heart rate variability, heart rate spectral power, cardiac and renal norepinephrine spillover) have been documented to bear a close and direct relationship with cardiovascular mortality, disease progression and arrhythmic events, including sudden death (46-51). Similar conclusions have been drawn by assessing the prognostic values of plasma norepinephrine or heart rate variability in the post-stroke phase following an acute myocardial infarction or in patients with vasospastic angina (52-55). Finally, dysfunction in sympathetic cardiovascular control has also been shown to have prognostic value in non-cardiovascular diseases such as diabetes mellitus and renal failure $(56,57)$, thereby documenting the clinical relevance of the neuroadrenergic overdrive in a broad spectrum of pathological states.

To date, the evidence supporting a role of sympathetic activation in metabolic syndrome appears to be less stringent than in the above-mentioned diseases. However, solid data support the notion that sympathetic neural mechanisms participate in the development and progression of metabolic syndromerelated target organ damage. One example is represented by left ventricular hypertrophy, whose occurrence has been shown to further worsen the already compromised survival of patients with metabolic syndrome (58). Studies performed "in vitro" have indeed shown that the growth of cardiomyocytes is under adrenergic influences (59). The "pro-hypertrophic" effects exerted by the sympathetic nervous system on myocardial tissue have been confirmed in animal studies showing that the systemic infusion of norepinephrine for several weeks, even when devoid of any pressor effect, triggers an increase in left ventricular mass (60). The evidence collected so far also extends to human beings. For example, it has been reported that in hypertensive patients a coronary arteriovenous difference in plasma norepinephrine is enhanced in the presence of left ventricular hypertrophy (61), indicating that the hypertrophic heart is associated with (and probably dependent on) an increased adrenergic cardiovascular drive. Furthermore, in hypertensive patients with echocardiographic evidence of left ventricular hypertrophy: 1) cardiac norepinephrine spillover is increased (13) and 2) sympathetic neural discharge, as quantified by the microneurographic approach, is enhanced (12). Finally, in conditions characterized by a marked sympathetic activation, such as in the hypertensive state complicated by end-stage renal failure, venous norepinephrine (i.e., an indirect marker of adrenergic drive) also displays an increase in its circulating blood levels (62). A further example is represented by arterial stiffness and vascular 
hypertrophy (63), given the evidence that sympathetic influences 1) modulate beat-to-beat the elastic properties of largeand medium-size arteries $(64), 2)$ participate at the development and/or progression of vascular structural alterations (65) and 3) promote the endothelial dysfunction and thus the occurrence of atherosclerotic vascular lesions (65).

Taken together, these findings support the notion that in the metabolic syndrome, as in other cardiovascular diseases, sympathetic overactivity has an adverse impact on both cardiovascular function and cardiovascular prognosis.

\section{Therapeutic Implications}

Although at present no specific guidelines for the treatment of the metabolic syndrome are available, there is a consensus among various scientific societies and organizations, including the European Task Force for Cardiovascular Prevention, the European Society of Hypertension and the American Heart Association, that specific pharmacologic and non-pharmacological interventions should be suggested when the values of the various variables clustering in the metabolic syndrome are clearly abnormal or even when they are in the "high-normal" range (66-68). This appears to be particularly the case for blood pressure values, whose cutoff values for initiating drug treatment are $\geq 130 / 85 \mathrm{mmHg}(68)$, i.e., clearly below the values indicated in uncomplicated essential hypertension. This may also be the case for resting tachycardia, which can be frequently detected in the metabolic syndrome $(58,68,69)$ and may represent an early marker of cardiovascular risk (70).

Based on the data discussed so far, sympathoinhibition appears to be a key goal of the therapeutic approach to the metabolic syndrome. Non-pharmacological lifestyle interventions commonly used in clinical practice and capable of improving both the hemodynamic and the metabolic profile, such as energy-restricted diet or physical training, have been clearly shown to exert sympathoinhibitory effects (71-73). Interestingly, this sympathetic deactivation is paralleled by a clearcut improvement in insulin resistance, a finding that once again documents the close relationships existing between the adrenergic and the metabolic function.

Sympathetic deactivation should also be one of the goals of pharmacological interventions employed in the metabolic syndrome, and particularly of antihypertensive drug treatment (74). While diuretics and $\beta$-blockers appear to be clearly contraindicated due to their unfavorable metabolic effects, the preferred antihypertensive drugs in the treatment of the disease appear to be calcium-antagonists, angiotensin-converting enzyme (ACE) inhibitors and angiotensin II antagonists (74). This is because these drugs 1) are effective in ensuring a blood pressure control over a 24-h period, 2) are lipid neutral, 3) are capable of improving insulin sensitivity and 4) exert neutral (calcium antagonists) or favorable (ACE inhibitors and angiotensin II receptor blockers) effects on sympathetic function (74). To these evidences should be added the finding that 1) new calcium channel blockers, such as cilnidipine and barnidipine, may exert inhibitory effects on adrenergic function (75), unlike "classic" compounds, and 2) new central sympatholitic agents, such as imidazoline $\mathrm{I}_{1}$, receptor agonists may trigger favorable metabolic and sympathoinhibitory effects (74). This recently developed class of drugs, which includes monoxidine and rilmenidine, exerts central sympathoinhibition by stimulating type I imidazoline receptors located in the rostro-ventromedullary region of the brain stem (76). Their clinical use in metabolic syndrome is supported by their favorable effects on insulin sensitivity, glucose tolerance and lipid profiles (76).

\section{Conclusions}

In recent years, the relationships among sympathetic dysfunction, metabolic syndrome and cardiovascular risk have engendered much interest. As a result, sympathetic overactivity is increasingly being considered a major target for therapeutic interventions aimed at reducing global cardiovascular risk.

\section{References}

1. Reaven GM, Lithell H, Landsberg L: Hypertension and associated metabolic abnormalities - the role of insulin resistance and the sympathoadrenal system. $N$ Engl $J$ Med 1996; 334: 374-381.

2. Morrison SF: Central pathways controlling brown adipose tissue thermogenesis. News Physiol Sci 2004; 19: 67-74.

3. Grassi G: Role of the sympathetic nervous system in human hypertension. J Hypertens 1998; 16: 1979-1987.

4. Grassi G, Mancia G: Neurogenic hypertension: is the enigma of its origin near the solution? Hypertension 2004; 43: $154-155$.

5. Amerena J, Julius S: The role of the autonomic nervous system in hypertension. Hypertens Res 1995; 18: 99-110.

6. Julius S, Krause L, Schork NJ, et al: Hyperkinetic borderline hypertension in Tecumseh, Michigan. Hypertension 1991; 9: 77-84.

7. Grassi G, Mancia G: The function of the autonomic nervous system in hypertension, in Bolis L, Licinio J, Govoni S (eds): Handbook of the Autonomic Nervous System. New York, Marcel Dekker, 2003, pp 425-438.

8. Grassi G, Vailati S, Bertinieri G, et al: Heart rate as marker of sympathetic activity. J Hypertens 1998; 16: 1635-1639.

9. Grassi G, Esler M: How to assess sympathetic activity in humans. J Hypertens 1999; 17: 719-734.

10. Grassi G, Cattaneo BM, Seravalle G, Lanfranchi A, Mancia G: Baroreflex control of sympathetic nerve activity in essential and secondary hypertension. Hypertension 1998; 31: $68-72$.

11. Grassi G, Seravalle G, Bertinieri G, et al: Sympathetic and reflex alterations in systo-diastolic and systolic hypertension of the elderly. J Hypertens 2000; 18: 587-593.

12. Greenwood JP, Scott EM, Stoker JB, Mary DA: Hypertensive left ventricular hypertrophy: relation to peripheral sympathetic drive. J Am Coll Cardiol 2001; 38: 1711-1717.

13. Schlaich MP, Kaye DM, Lambert E, Sommerville M, 
Socratous F, Esler MD: Relation between cardiac sympathetic activity and hypertensive left ventricular hypertrophy. Circulation 2003; 108: 560-565.

14. Esler M, Ferrier C, Lambert G, Eisenhofer G, Cox H, Jennings G: Biochemical evidence of sympathetic hyperactivity in human hypertension. Hypertension 1991; 17: S29S35.

15. Ferrier C, Esler MD, Eisenhofer G, et al: Increased norepinephrine spillover into the jugular veins in essential hypertension. Hypertension 1992; 19: 62-69.

16. Young JB, Macdonald IA: Sympathoadrenal activity in human obesity: heterogeneity of findings since 1980. Int $J$ Obes Relat Metab Disord 1992; 16: 959-967.

17. Troisi RJ, Weiss ST, Parker DR, Sparrow D, Young JB, Landsberg L: Relation of obesity and diet to sympathetic nervous system activity. Hypertension 1991; 17: 669-677.

18. Grassi G, Seravalle G, Cattaneo BM, et al: Sympathetic activation in obese normotensive subjects. Hypertension 1995; 25: 560-563.

19. Vaz M, Jennings G, Turner A, Cox H, Lambert G, Esler M: Regional sympathetic nervous activity and oxygen consumption in obese normotensive human subjects. Circulation 1997; 96: 3423-3429.

20. Grassi G, Dell'Oro R, Facchini A, Quarti Trevano F, Bolla GB, Mancia G: Effect of central and peripheral body fat distribution on sympathetic and baroreflex function in obese normotensives. J Hypertens 2004; 22: 2363-2369.

21. Alvarez GE, Ballard TP, Beske SD, Davy KP: Subcutaneous obesity is not associated with sympathetic neural activation. Am J Physiol 2004; 287: H414-H418.

22. Narkiewicz K, van de Borne PJ, Cooley RL, Dyken ME, Somers VK: Sympathetic activity in obese subjects with and without obstructive sleep apnea. Circulation 1998; 98: $772-776$.

23. Grassi G, Facchini A, Trevano FQ, et al: Obstructive sleep apnea-dependent and-independent adrenergic activation in obesity. Hypertension 2005; 46: 321-325.

24. Grassi G, Seravalle G, Dell'Oro R, Turri C, Bolla GB, Mancia G: Adrenergic and reflex abnormalities in obesityrelated hypertension. Hypertension 2000; 36: 538-542.

25. Brunner EJ, Hemingway H, Walker BR, et al: Adrenocortical, autonomic, and inflammatory causes of the metabolic syndrome: nested case-control study. Circulation 2002; 106: 2659-2665.

26. Straznicky NE, Lambert EA, Lambert GW, Masuo K, Esler MD, Nestel PJ: Effects of dietary weight loss on sympathetic activity and cardiac risk factors associated with the metabolic syndrome. J Clin Endocrinol Metab 2005; 90: 5998-6005.

27. Huggett RJ, Burns J, Mackintosh AF, Mary DA: Sympathetic neural activation in nondiabetic metabolic syndrome and its further augmentation by hypertension. Hypertension 2004; 44: 847-852.

28. Grassi G, Dell'Oro R, Quarti-Trevano F, et al: Neuroadrenergic and reflex abnormalities in patients with metabolic syndrome. Diabetologia 2005; 48: 1359-1365.

29. Arenare F, Quarti Trevano F, Dell'Oro R, et al: Metabolic syndrome potentiates the sympathetic and baroreflex dysfunction of heart failure state. J Hypertens 2006; 24: S6 (Abstract).
30. Grassi G, Giannattasio C, Cleroux J, et al: Cardiopulmonary reflex before and after regression of left ventricular hypertrophy in essential hypertension. Hypertension 1988; 12: 227-237.

31. Narkiewicz K, van de Borne PJ, Pesek CA, Dyken ME, Montano N, Somers VK: Selective potentiation of peripheral chemoreflex sensitivity in obstructive sleep apnea. Circulation 1999; 99: 1183-1189.

32. Landsberg L: Obesity and the insulin resistance syndrome. Hypertens Res 1996; 19: S51-S55.

33. Egan BM: Insulin resistance and the sympathetic nervous system. Curr Hypertens Rep 2003; 5: 247-254.

34. Anderson EA, Balon TW, Hoffman RP, Sinkey CA, Mark AL: Insulin increases sympathetic activity but not blood pressure in borderline hypertensive humans. Hypertension 1992; 19: 621-627.

35. Scherrer U, Sartori C: Insulin as a vascular and sympathoexcitatory hormone: implications for blood pressure regulation, insulin sensitivity, and cardiovascular morbidity. Circulation 1997; 96: 4104-4113.

36. Grassi G, Seravalle G, Quarti Trevano F, et al: Potentiating effects of the metabolic syndrome on the sympathetic abnormalities characterizing human obesity. $J$ Hypertens 2005; 23: S266 (Abstract).

37. Jamerson KA, Julius S, Gudbrandsson T, Andersson O, Brant DO: Reflex sympathetic activation induces acute insulin resistance in the human forearm. Hypertension 1993; 21: 618-623.

38. Li YF, Wang W, Mayhan WG, Patel KP: Angiotensinmediated increase in renal sympathetic nerve discharge within the PVN: role of nitric oxide. Am J Physiol Regul Integr Comp Physiol 2006; 290: R1035-R1043.

39. Taddei S, Grassi G: Angiotensin II as the link between nitric oxide and neuroadrenergic function. $J$ Hypertens 2005; 23: 935-937.

40. Grassi G: Renin-angiotensin-sympathetic crosstalks in hypertension: reappraising the relevance of peripheral interactions. J Hypertens 2001; 19: 1713-1716.

41. Grassi G, Seravalle G, Dell'Oro R, et al: CROSS Study. Comparative effects of candesartan and hydrochlorothiazide on blood pressure, insulin sensitivity, and sympathetic drive in obese hypertensive individuals: results of the CROSS study. J Hypertens 2003; 21: 1761-1769.

42. Klein IH, Ligtenberg G, Oey PL, Koomans HA, Blankestijn PJ: Enalapril and losartan reduce sympathetic hyperactivity in patients with chronic renal failure. $J \mathrm{Am}$ Soc Nephrol 2003; 14: 425-430.

43. Grassi G, Cattaneo BM, Seravalle G, et al: Effects of chronic ACE inhibition on sympathetic nerve traffic and baroreflex control of circulation in heart failure. Circulation 1997; 96: 1173-1179.

44. Grassi G: Leptin, sympathetic nervous system, and baroreflex function. Curr Hypertens Rep 2004; 6: 236-240.

45. Rahmouni K, Correia ML, Haynes WG, Mark AL: Obesityassociated hypertension: new insights into mechanisms. Hypertension 2005; 45: 9-14.

46. Cohn JN, Levine TB, Olivari MT, et al: Plasma norepinephrine as a guide to prognosis in patients with chronic congestive heart failure. $N$ Engl J Med 1984; 311: 819-823.

47. Rouleau JL, Packer M, Moye L, et al: Prognostic value of 
neurohumoral activation in patients with an acute myocardial infarction: effect of captopril. J Am Coll Cardiol 1994; 24: 583-591.

48. Brunner-La Rocca HP, Esler MD, Jennings GL, Kaye DM: Effect of cardiac sympathetic nervous activity on mode of death in congestive heart failure. Eur Heart $J$ 2001; 22: 1136-1143.

49. Gerson MC, McGuire N, Wagoner LE: Sympathetic nervous system function as measured by I-123 metaiodobenzylguanidine predicts transplant-free survival in heart failure patients with idiopathic dilated cardiomyopathy. $J$ Card Fail 2003; 9: 384-391.

50. Bristow MR, Krause-Steinrauf H, Nuzzo R, et al: Effect of baseline or changes in adrenergic activity on clinical outcomes in the beta-blocker evaluation of survival trial. Circulation 2004; 110: 1437-1442.

51. Petersson M, Friberg $P$, Eisenhofer G, Lambert G, Rundqvist B: Long-term outcome in relation to renal sympathetic activity in patients with chronic heart failure. Eur Heart J 2005; 26: 906-913.

52. Sander D, Winbeck K, Klingelhofer J, Etgen T, Conrad B: Prognostic relevance of pathological sympathetic activation after acute thromboembolic stroke. Neurology 2001; 57: 833-838.

53. Copie X, Hnatkova K, Staunton A, Fei L, Camm AJ, Malik $\mathrm{M}$ : Predictive power of increased heart rate versus depressed left ventricular ejection fraction and heart rate variability for risk stratification after myocardial infarction. Results of a two-year follow-up study. J Am Coll Cardiol 1996; 27: 270-276.

54. La Rovere MT, Bigger JT Jr, Marcus FI, Mortara A, Schwartz PJ, ATRAMI (Autonomic Tone and Reflexes after Myocardial Infarction) Investigators: Baroreflex sensitivity and heart-rate variability in prediction of total cardiac mortality after myocardial infarction. Lancet 1998; 351: 478-484.

55. Benedict CR, Shelton B, Johnstone DE, SOLVD Investigators: Prognostic significance of plasma norepinephrine in patients with asymptomatic left ventricular dysfunction. Circulation 1996; 94: 690-697.

56. Endo A, Kinugawa T, Ogino K, et al: Cardiac and plasma catecholamine responses to exercise in patients with type 2 diabetes: prognostic implications for cardiac-cerebrovascular events. Am J Med Sci 2000; 320: 24-30.

57. Zoccali C, Mallamaci F, Parlongo S, et al: Plasma norepinephrine predicts survival and incident cardiovascular events in patients with end-stage renal disease. Circulation 2002; 105: 1354-1359.

58. Mancia G, Sega R, Cesana GC, et al: Metabolic syndrome in PAMELA population: prevalence, prognostic value, outof-office blood pressures and cardiac damage. $J$ Hypertens 2005; 23: S134 (Abstract).

59. Sen S, Tarazi RC, Khairallah PA, Bumpus FM: Cardiac hypertrophy in spontaneously hypertensive rats. Circ Res 1974; 35: 775-781.

60. Patel MB, Stewart JM, Loud AV, et al: Altered function and structure of the heart in dogs with chronic elevation in plasma norepinephrine. Circulation 1991; 84: 2091-2100.

61. Kelm M, Schafer S, Mingers S, et al: Left ventricular mass is linked to cardiac noradrenaline in normotensive and hypertensive patients. J Hypertens 1996; 14: 1357-1364.

62. Zoccali C, Mallamaci F, Tripepi G, et al: Norepinephrine and concentric hypertrophy in patients with end-stage renal disease. Hypertension 2002; 40: 41-46.

63. Seo HS, Kang TS, Park S, et al: Insulin resistance is associated with arterial stiffness in nondiabetic hypertensives independent of metabolic status. Hypertens Res 2005; 28: 945-951.

64. Grassi G, Giannattasio C, Failla M, et al: Sympathetic modulation of radial artery compliance in congestive heart failure. Hypertension 1995; 26: 348-354.

65. Grassi G, Giannattasio C: Obesity and vascular stiffness: when body fat has an adverse impact on arterial dynamics. $J$ Hypertens 2005; 23: 1789-1791.

66. De Backer G, Ambrosioni E, Borch-Johnsen K, et al: European guidelines on cardiovascular disease prevention in clinical practice. Third Joint Task Force of European and Other Societies on Cardiovascular Disease Prevention in Clinical Practice. Eur Heart J 2003; 24: 1601-1610.

67. European Society of Hypertension-European Society of Cardiology Guidelines Committee: 2003 European Society of Hypertension-European Society of Cardiology guidelines for the management of arterial hypertension. J Hypertens 2003; 21: 1011-1053.

68. Grundy SM, Cleeman JI, Daniels SR, et al: Diagnosis and management of the metabolic syndrome: an American Heart Association/National Heart, Lung, and Blood Institute Scientific Statement. Circulation 2005; 112: 2735 2752.

69. Takeuchi H, Saitoh S, Takagi S, et al: Metabolic syndrome and cardiac disease in Japanese men: applicability of the concept of metabolic syndrome defined by the National Cholesterol Education Program-Adult Treatment Panel III to Japanese men - the Tanno and Sobetsu Study. Hypertens Res 2005; 28: 203-208.

70. Palatini P, Benetos A, Grassi G, et al: Identification and management of the hypertensive patient with elevated heart rate: statement of a European Society of Hypertension Consensus Meeting. J Hypertens 2006; 24: 603-610.

71. Grassi G, Seravalle G, Colombo M, et al: Body weight reduction, sympathetic nerve traffic, and arterial baroreflex in obese normotensive humans. Circulation 1998; 97: 2037-2042.

72. Grassi G, Seravalle G, Calhoun DA, Mancia G: Physical training and baroreceptor control of sympathetic nerve activity in humans. Hypertension 1994; 23: 294-301.

73. Iwane M, Arita M, Tomimoto S, et al: Walking 10,000 steps/day or more reduces blood pressure and sympathetic nerve activity in mild essential hypertension. Hypertens Res 2000; 23: 573-580.

74. Grassi G: Counteracting the sympathetic nervous system in essential hypertension. Curr Opin Nephrol Hypertens 2004; 13: 513-519.

75. Grassi G: Neuroadrenergic effects of calcium channel blockers: a developing concept. J Hypertens 2004; 22: 887888.

76. van Zwieten PA: Centrally acting imidazoline $I_{1}-$ receptor agonists: do they have a place in the management of hypertension? Am J Cardiovasc Drugs 2001; 1: 321-326. 